\title{
Effect of Low-Frequency Non-Invasive Therapeutic Ultrasound on Attenuation of Cardiac Fibrosis Early After Myocardial Infarction in Rats
}

\section{Elias Daud}

Galilee Medical Center

Offir Ertracht ( $\nabla$ offire@gmc.gov.il )

Galilee Medical Center https://orcid.org/0000-0002-2249-9194

\section{Gassan Moady}

Galilee Medical Center

\section{Jamal Awad}

Galilee Medical Center

\section{Aviad Rotenberg}

Galilee Medical Center

Tal Reuveni

Galilee Medical Center

\section{Shaul Atar}

Galilee Medical Center

\section{Research Article}

Keywords: Myocardial infarction, therapeutic ultrasound, collagen deposition, Transforming Growth Factor $\beta 1$, Smad3

Posted Date: January 21st, 2022

DOI: https://doi.org/10.21203/rs.3.rs-1265416/v1

License: (a) (1) This work is licensed under a Creative Commons Attribution 4.0 International License. Read Full License 


\section{Abstract}

Background: Myocardial infarction (MI) results in collagen deposition leading to left ventricular dysfunction. Therapeutic ultrasound (TUS) has proven anti-fibrotic effects on various tissues. We hypothesized that noninvasive TUS will attenuate fibrosis by downregulation of the transforming growth factor $\beta 1$ (TGF- $\beta 1$ )/ small mother against decapentaplegic $3(\operatorname{smad} 3)$ pathway in a rat model of $\mathrm{MI}$.

Methods: Rats underwent MI induction procedure after performing baseline cardiac echocardiography. Subsequently, we treated the rats with either daily spironolactone (a known anti-fibrotic agent), TUS (at $1 \mathrm{MHz}$ frequency and $0.5 \mathrm{~W} / \mathrm{cm}^{2}$ intensity, 3 times a week) or their combination (spironolactone + TUS). Control rats were not treated. We re-evaluated cardiac function at 2 and 4 weeks after treatment initiation (before sacrifice), then performed histological and biochemical evaluation of fibrosis.

Results: Any treatment combination attenuated fibrosis, as seen in histology and by chemical analysis, and reduced TGF- $\beta 1$ expression. Specifically, collagen volume fractions were $16.0 \pm 9.6 \%, 5.0 \pm 3.2 \%, 7.2 \pm 1.0 \%$ and $1.6 \pm 0.9 \%$, and TGF- $\beta 1$ expression were $3.34 \pm 0.36 \%, 0.77 \pm 0.37 \%, 0.59 \pm 0.25 \%$ and $0.39 \pm 0.16 \%$, in the control (untreated group), spironolactone. TUS and spironolactone + TUS groups, respectively $(P<0.05$ vs. control for all). Yet, only spironolactone treatments affected also smad3 expression, and cardiac physiology, i.e., final ejection fraction were $36.0 \pm 2.5 \%, 50.1 \pm 2.3 \%, 45.2 \pm 2.5 \%$ and $54.0 \pm 2.7 \%$ in the control (untreated group), spironolactone. TUS and spironolactone + TUS groups, respectively $(\mathrm{P}<0.05 \mathrm{vs}$. control for spironolactone and spironolactone + TUS only).

Conclusion: Post MI, TUS reduces myocardial collagen, fibrosis and TGF- $\beta 1$ content. Only with the addition of spironolactone cardiac function was preserved. Further research is needed in order to refine and upgrade TUS to affect cardiac physiology early post MI.

\section{Introduction}

Myocardial infarction (MI) is a major cause of collagen deposition and myocardial fibrosis, leading to ventricular remodeling and dysfunction [1]. Inhibiting post-MI collagen deposition may attenuate left ventricular (LV) remodeling, prevent heart failure and thus decrease morbidity and mortality.

LV remodeling is affected mainly by the renin-angiotensin-aldosterone system (RAAS). It was shown that aldosterone plays a major role in LV remodeling [2], as high aldosterone levels were associated with cardiac hypertrophy and fibrosis in rats following $\mathrm{MI}[3]$ or systemic hypertension [4, 5]. Accordingly, the pro-fibrotic effect of aldosterone, through collagen deposition, possibly facilitates the occurrence of the clinical syndrome of heart failure. Additionally, it was found that aldosterone inhibitors attenuate ventricular changes due to myocardial injury especially post MI [1]. Mill et al. showed that the aldosterone inhibitor, spironolactone, when administered shortly after $\mathrm{MI}$, mitigated cardiac remodeling and the development of fibrosis [1]. Lately, it was proven that the effect of aldosterone on fibrosis is partly through the activation of the transforming growth factor beta (TGF- $\beta$ ) pathway $[6,7]$. Further, the involvement of pro-inflammatory cytokines such as TGF- $\beta 1$ and its down-stream effector small mother against decapentaplegic 3 (smad3), as mediators of cardiac tissue fibrosis after injury, inflammation and apoptosis was established [8]. Kang et al. showed in an in vitro set-up, that collagen deposition reduction may involve the inhibition of the TGF- $\beta 1 /$ smad3 pathway [9]. Lately, we have 
shown in an in vivo set-up that empagliflozin attenuates TGF- $\beta 1 /$ smad3 expression, and concomitantly, affected collagen deposition in non-diabetic rats post MI [10], thus, establishing further the involvement of this pathway in fibrosis.

In recent years, ultrasound has been used as a therapeutic tool for various diseases ranging from kidney stones (lithotripsy) [11] to cancer [12]. The effect of therapeutic ultrasound (TUS) on tissue perfusion, as well as denovo angiogenesis was also established $[13,14]$. Additionally, we showed that specific TUS profiles may affect nitric oxide synthase (NOS) expression, while other studies showed that TUS increases nitric oxide (NO) production $[15,16]$. Another study related an anti-fibrotic effect to sonodynamic therapy (SDT) [17]. Specifically, this effect was associated with activation of the cytotoxic reagent 5-aminolevulinic acid (ALA) in rat cardiac fibroblasts [17]. Further, it was also shown that ultrasound therapy affects collagen fibers organization in other tissues such as rats' tendons [18]. Finally, Rossato et al. showed that high intensity focused ultrasound modulated pro- and anti-inflammatory cytokines such as tumor necrosis factor $a$ and interleukin 6 and 10 in skeletal muscle of rats with heart failure after ligation of the left coronary artery [8].

We hypothesized that early application of non-invasive TUS, with and without spironolactone, will attenuate collagen deposition post $\mathrm{MI}$ and affect LV remodeling and function by reducing TGF- $\beta 1$ myocardial content and its associated protein smad3.

\section{Material And Methods}

\section{Animals}

All animal experiments were conducted according to the institutional animal ethical committee guidelines, which conform to the Guide for the Care and Use of Laboratory Animals published by the US National Institutes of Health (Eighth edition 2011) (Ethics 09-01-2017). We used 250-300 gr. Sprague-Dawley rats (Harlan Laboratories Ltd, Jerusalem, Israel), which were maintained at a constant temperature and relative humidity under a regular light-dark schedule (12h:12h), fed with normal rodent diet and with tap water ad libitum.

\section{Study design}

All animals underwent baseline cardiac function evaluation by echocardiography. Subsequently, the animals underwent Ml procedure. Then, we divided the animals into 4 experimental groups $(n=5-6)$. The first group of untreated rats composed the control group. The second group received spironolactone (a known anti-fibrotic agent). The third group received TUS and the fourth group received spironolactone + TUS. Therapy was administered for 4 consecutive weeks.

Cardiac function was re-evaluated 2 weeks after treatment initiation and at the end of the experiments, before sacrifice (4 weeks from study initiation). After euthanasia, we excised the heart and preserved it for additional biochemical and histological analyses.

\section{Echocardiography}

Echocardiography scans were taken as previously described [19]. Briefly, under light sedation with $29 \mathrm{mg} / \mathrm{kg}$ ketamine and $4.3 \mathrm{mg} / \mathrm{kg}$ xylazine, the rats were placed in a left decubitus position and scanned via a commercial echo-scanner (Vivid $i$. frequency $9 \mathrm{MHz}$, depth $2.5 \mathrm{~cm}$ and frame rate $315 \mathrm{frames} / \mathrm{sec}$ ). We scanned 
2 parasternal short axis sections at apical (AP) and papillary muscle (PM) levels. Two-dimensional (2D) echocardiography scans took place at baseline before MI, 2 weeks after surgery and at the end of the experiments (4 weeks after $\mathrm{MI})$.

The echocardiography analysis included structural parameters such ventricular diameters and cross sectional areas at diastole and systole. For ventricular function assessment, 3 parameters were measured: (1) Fractional shortening (FS) which takes into consideration a 2D cross-section of the heart. Specifically, in the M-mode cines, the left ventricle (LV) intra-ventricular diameters were measured at systole and diastole (LVIDs and LVIDd, respectively), and FS was calculated as follows: FS = (LVIDd-LVIDs)/LVIDd; (2) Fractional area change (FAC) was calculated as the change in LV area during cardiac cycle. Particularly, LV end diastolic area (LVEDA) and LV end systolic area (LVESA) are measured in 2D cines and FAC were calculated as follows: FAC = (LVEDALVESA)/ LVEDA; (3) Ejection fraction (EF) was determined by the Vivid $i$ LV function software. The echocardiography analysis was performed for each level (AP and PM) by itself, as generally, the cardiac damage may affect distinct levels differently.

\section{Myocardial Infarction}

Rats underwent MI procedure, as previously described by Daud et al. [10]. Briefly, under deep anesthesia with a mixture of $87 \mathrm{mg} / \mathrm{kg}$ ketamine and $13 \mathrm{mg} / \mathrm{kg}$ xylazine rats were intubated and ventilated at a rate of 80-90 breaths $x$ min $^{-1}$ and 1 to $2 \mathrm{ml} \times(100 \mathrm{gr})^{-1}$ tidal volume. Using intercostal space left thoracotomy, the chest was opened and the pericardial sac was dissected. A stich was placed through myocardium at a slightly greater depth than the perceived level of the left anterior descending (LAD) artery. The suture was tightened for complete LAD occlusion, the chest was closed, the skin - stitched, and the rat was placed in its cage for recovery.

\section{Spironolactone treatment}

Spironolactone $20 \mathrm{mg} / \mathrm{kg} /$ day dissolved in paraffin oil (Sigma chemicals, St Louis, MO, USA) was administered daily by sub-cutaneous injection [1, 4, 20]. Rats were weighted weekly to adjust dosage. The control groups got equivalent volume of oil.

\section{Therapeutic ultrasound}

We applied TUS to the anterior chest wall. The TUS profile is characterized by its duration (3 minutes), intensity $(1 \mathrm{MHz})$ and frequency $\left(0.5 \mathrm{~W} / \mathrm{cm}^{2}\right)$ [18], using an ultrasound transducer (V106-SB, Olympus, Waltham, MA, USA) with an effective radiating area of $127 \mathrm{~mm}^{2}$. The non-TUS groups were manipulated in the same way but with US equipment turned off.

\section{Animal sacrifice and organ harvesting}

After the last measurement, the animals were euthanized by high anesthetic dosage. We dissected the cardiac muscle transversally at the PM level. The cardiac apex was fixated in $4 \%$ buffered formaldehyde and then embedded in paraffin for histology, while the base was flash frozen in liquid nitrogen and preserved in $-80^{\circ} \mathrm{C}$ for chemical and molecular analyses.

\section{Histology}


The paraffin-embedded apex was sectioned into $5 \mu \mathrm{m}$ using a microtome and was stained with Hematoxylin and Eosin (H\&E), Tri-chrome Masson's and Picro-Sirius red. All hearts were evaluated for their structural parameters $(n=5-6)$. From each animal, its H\&E section was photographed. Total Cross-sectional area, LV crosssectional area, LV cavity area, and septal and anterior wall widths were measured using the ImageJ freeware ( $\mathrm{NIH}$, Bethesda, Md, USA).

\section{Tri-chrome Masson's stain}

Tri-chrome Masson's stain was used for studying connective tissue, muscle and collagen. Tri-chrome Masson's stain was used primarily for distinguishing collagen from muscle tissue. Briefly, after deparafinization and rehydration, slides were placed in $56^{\circ} \mathrm{C}$ preheated Bouin's solution for 15 minutes. Then they were cooled in tap water $\left(18-26^{\circ} \mathrm{C}\right)$ and washed to remove excess Bouin's solution. Afterwards we stained the slides in Weigert's iron Hematoxylin solution for 5 minutes, and then washed in running tap water for 5 minutes followed by staining in Biebrich Scarlet-Acid Fucshin for 5 minutes. Subsequently, we rinsed the slides in deionized water, and placed them in phosphotungstic/phosphomolybdic acid solution for 5 minutes. Finally, slides were placed in Aniline Blue solution for 5 minutes and then in 1\% Acetic Acid for 2 minutes. Solutions were then discarded, slides dehydrated through alcohol, cleared in xylene and mounted.

Collagen area was measured in scar and remote regions under microscope (Nikon Eclipse Ci-L, Nikon Corporation, Tokyo, Japan) using the NIS Elements software (NIS Elements 4.0, Nikon Corporation, Tokyo, Japan). For each animal, total 5 fields of remote area and 5 fields of scar area were photographed (x100 magnification). In each, picture the collagen (blue) area was measured and so was the total tissue area, finally, the \% of collagen was calculated by dividing collagen area by total tissue area in the picture to give the collagen volume fraction (CVF). Each groups' data was averaged. The data was obtained blindly.

\section{Picro-Sirius red stain}

This stain demonstrates collagen and muscle. Briefly, after deparafinization and rehydration, we rinsed the slides in distilled water and stained nuclei with Weigert's Hematoxylin solution for 8 minutes. Then, we placed the slides in Picro-Sirius red stain for 60 minutes. Next, we rinsed in 2 changes of $0.5 \%$ Acetic acid water followed by quick dehydration through 3 changes of absolute alcohol. Finally, slides were mounted with coverslip using a permanent mounting medium.

Collagen area was measured in scar and remote regions under microscope (Nikon Eclipse Ci-L, Nikon Corporation, Tokyo, Japan) using the NIS Elements software (NIS Elements 4.0, Nikon Corporation, Tokyo, Japan). Analysis of data and CVF was estimated as for the tri-chrome Masson's stain, with the exception that the collagen area was the red colored tissue.

\section{Collagen concentration measurement}

Collagen concentration was estimated in the LV muscle samples by measuring the hydroxyproline concentration [21]. Briefly, frozen cardiac samples were minced and then hydrolyzed at $120^{\circ} \mathrm{C}$ for 20 minutes in $1 \mathrm{~N} \mathrm{HCl}, 450 \mu$ l of Chloramine-T was added to the hydrolysate, oxidation continued for 25 minutes in RT. We added $500 \mu \mathrm{l}$ of Ehrlich's aldehyde reagent to the samples and incubated at $65^{\circ} \mathrm{C}$ for 20 minutes. Optical density was measured at $550 \mathrm{~nm}$, pure rat tail collagen was used for calibration of the Optical density, hence, the results are expressed as $\mu \mathrm{g}$, all samples were measured in duplicates. 


\section{Immunohistochemical staining}

Sections of myocardial tissue underwent deparafinization and rehydration. Antigen retrieval was achieved by heating the specimens for $10 \mathrm{~min}$ at $94^{\circ} \mathrm{C}$ in citric acid buffer $(0.01 \mathrm{~mol} / \mathrm{l} ; \mathrm{pH} 6.0)$. Sections were then incubated with 3\% hydrogen peroxide at RT for 20 min to inactivate endogenous peroxidase activity. After rinsing with PBS buffer (pH 7.2), primary antibodies against TGF- $\beta 1$ (1:200; cat. no. Ab92486; abcam Cambridge, MA, USA) or smad3 (1:200; cat. no. Ab40854; abcam Cambridge, MA, USA), were added and incubated at $4^{\circ} \mathrm{C}$ overnight. Following further rinsing, anti-rabbit horseradish peroxidase-conjugated secondary antibody (1:1,000; cat. no. D13-6; GBI Labs, Bothell, WA, USA) was added and incubated at RT for $15 \mathrm{~min}$. Samples were subsequently incubated with DAB solution at RT until color development. A total of 5 different fields of view were randomly selected under a light microscope with magnification of $\times 400$. The average absorbance of TGF- $\beta 1$, was analyzed using the NIS-Elements Software BR analysis system version 4.10.00 (Nikon Corporation).

\section{Statistical analysis}

Data presented as mean $\pm S D$, comparisons between groups were performed by 2-way analysis of variance (ANOVA) with repeated measures, in which the treatment and the time point were the independent variables. Final data were compared between groups using 1-way ANOVA in which the treatment group was the independent variable. Whenever the ANOVA was significant, a multiple comparison was made using the HolmSidak as post-hoc test. P value of $<0.05$ was considered significant.

\section{Results}

\section{Fibrosis}

In the current research, we measured fibrosis using three distinct methods. Collectively, all three methods indicate a reduction of collagen content in the tissues taken from treated rats vs. the untreated controls. The fibrotic process, as seen by collagen deposition was significantly attenuated, at both scar area, as well as remote from the scar areas (referred as "remote areas") of the cardiac muscle (Fig. 1). Specifically, in the trichrome Masson's stain collagen is highly abundant in the scar tissues with almost no healthy muscle tissue (Fig. 1A). Collagen deposition was prevented by TUS (Fig. 1B), spironolactone (Fig. 1C) and the dual treatment (Fig. 1D). The abundance of collagen in remote areas at the untreated rats (Fig. 1E) looks higher than in the treated rats (Fig. 1F-H). Specimen stained with Picro-sirius red show similar distribution of collagen as seen in the Tri-chrome Masson's stain. Specifically, one may see high collagen expression in the scar of untreated rats' muscle (Fig. 1I), and lower collagen stains in treated specimen (Fig. 1J-L). Concomitantly, collagen distribution at remote areas follows the same pattern, i.e., high abundance in the untreated rats, and lower collagen level in treated rats (Fig. $1 \mathrm{M}$ and Fig. 1N-P, respectively).

CVF was further quantified in the histological specimen stained with either Tri-chrome Masson's or Picro-Sirius red, in the two regions of the heart, i.e., the scar itself and at remote cardiac areas. The control group's scar contained $75.3 \pm 5 \%$ collagen as measured by the Tri-chrome Masson's stain and $71.1 \pm 7.2 \%$ using the PicroSirius red stain (Fig. 2A-B). The spironolactone treatment, as expected, reduced scar CVF to $36.3 \pm 10.4 \%$ and $45.0 \pm 8.0 \%$ for Tri-chrome Masson's staining and the Picro-Sirius red staining, respectively $(\mathrm{P}<0.001$ vs. control) (Fig. 2A-B). TUS did not decrease the scar's CVF significantly as seen by the Tri-chrome Masson's staining 
(50.2 \pm 7.6\%, P>0.05 vs. control), yet a significant CVF decrease was found in the Picro-Sirius red staining (59.5 $\pm 4.7 \%, \mathrm{P}<0.05 \mathrm{vs}$. control). The spironolactone + TUS reduced the scar's CVF to $42.0 \pm 8.6 \%$ and $48.4 \pm 9.6 \%$ in the Tri-chrome Masson's and Picro-Sirius red, respectively ( $\mathrm{P}<0.001$ vs. control, for both) (Fig. 2A-B). Interestingly, the CVF in the TUS only group was higher than each of the other treatment groups, i.e., spironolactone alone and spironolactone + TUS (Fig. $2 A-B, P<0.05$, for both).

In the remote areas of the scar, the effect of the different treatments was more pronounced. Accordingly, in the control group, the Tri-chrome Masson's and Picro-Sirius red staining showed $24.7 \pm 8.6 \%$ and $16.0 \pm 9.6 \%$ CVF, respectively. Spironolactone treatment significantly reduced remote CVF to $3.4 \pm 1.5 \%(P<0.001$ vs. control) and $5.0 \pm 3.2 \%$ ( $P<0.01$ vs. control), respectively, for the two stains (Fig. 2C-D). The TUS treatment decreased CVF as well to $6.5 \pm 3.8 \%$ ( $\mathrm{P}<0.001$ vs. control) and $7.2 \pm 1.0 \%$ ( $\mathrm{P}<0.05$ vs. control) for these two stains, respectively (Fig. 2C-D). The spironolactone + TUS showed a positive effect in reducing CVF at the remote cardiac muscle as well. Specifically, the CVF was $1.9 \pm 0.9 \%$ and $1.6 \pm 0.9 \%$ in the Tri-chrome Masson's and the Picro-Sirius red staining, respectively $(P<0.001$ vs. control for both, Fig. 2C-D).

\section{Hydroxyproline analysis}

Hydroxyproline concentration, as a measure of collagen content is depicted in Fig. 3. The results indicate collagen at the scar and at remote sections of the cardiac muscle. Hydroxyproline concentration was reduced in scar as well as in remote regions in all 3 treated groups compared to the control group. However, statistical significance was found only by the TUS treatment $v s$. the control group $(P<0.05)$ at the scar region (Fig. $3 A)$.

\section{Immunohistology}

At the aim of establishing the effect of TUS on the fibrotic mechanism, we studied the cardiac muscle for its TGF- $\beta 1$ and smad3 expression, using immunohistochemical staining. The differences are apparent between the control group and the other therapeutic groups (Fig. 4). Fig. 4A depicts control specimen which is characterized with rather high TGF- $\beta 1$ expression, a reduced expression of TGF- $\beta 1$ is noted in all treated groups (Fig. 4B and 4C), with the lowest expression level in the spironolactone + TUS group (Fig. 4D). The smad3 seems to be affected differently, sill the highest expression is found in the control specimen, yet its expression seems to be reduced in both the spironolactone treated groups (spironolactone and spironolactone + TUS, Fig. $4 \mathrm{I}$ and $4 \mathrm{~J}$ ), but not in the TUS alone group (Fig. 4H). The measurements indicate that in the control group TGF- $\beta 1$ occupied $3.34 \pm 0.36 \%$ of the tissue's surface (Fig. 4F). In the spironolactone group, TUS group and spironolactone + TUS group, a reduction of TGF- $\beta 1$ expression was found, i.e., $0.77 \pm 0.37 \%, 0.59 \pm 0.25 \%$ and $0.39 \pm 0.16 \%$, respectively (Fig. 4F, $\mathrm{P}<0.001 \mathrm{vs}$. control for all). Smad3 was found to be highly expressed in the control group occupying $13.9 \pm 6.1 \%$ of the area. Following spironolactone or TUS treatments, the smad3 expression was $7.3 \pm 3.6 \%$ ( $P<0.01$ vs. control) and 18.2 $\pm 10.6 \%(P>0.05$ vs. control, respectively (Fig. $4 \mathrm{~L})$. However, by the spironolactone + TUS treatment, smad3 was reduced to $5.6 \pm 6.4 \%(P<0.05$ vs. control and $\mathrm{P}<0.01$ vs. TUS). Collectively, the results indicate that both treatments affected TGF- $\beta 1$, but not all treatments affect its downstream effector - smad3.

\section{Physiology}

Finally, in order to assess the physiological efficacy of the treatments, we have evaluated gross physiological parameters, such as body weight, heart weight and cardiac echocardiography. Twenty-three rats underwent the 
surgical procedure, two weeks after induction of $\mathrm{MI}$, the rats had attenuated cardiac function with reduced $\mathrm{EF}$ $(<50 \%)$, At sacrifice, we observed an obvious a significant scar region, which was also evident in H\&E in histology. Rat's initial body weight was $235 \pm 7 \mathrm{gr}$., animals of all experimental groups gained weight equally throughout the experiment, reaching $337 \pm 8$ gr., $353 \pm 8$ gr., $334 \pm 7$ gr. and $341 \pm 8$ gr. for the control, spironolactone, TUS and the spironolactone + TUS group, respectively $(P>0.05)$. Further, we found no difference in the heart weight, or the heart to body weight ratio.

\section{Echocardiography}

Detailed echocardiography data are summarized in supplementary Tables s1-s3. At baseline, cardiac structure and function were typical of healthy young rats: AP LVSEA was $14.5 \pm 3.2 \mathrm{~mm}^{2}$, LVEDA $30.5 \pm 3.4 \mathrm{~mm}^{2}$ and EF of $70.0 \pm 2.4 \%$ (Table s1). Fig. 5 depicts 2 cardiac functional parameters (FS and EF) as a function of time, treatment and their interaction on the parameters are best illustrated in the figure. Specifically, all measurements of all groups taken at 2 and 4 weeks are significantly lower than basal measurement $(P<0.001)$. Further, as the AP level is the cardiac territory which is most affected by the Ml procedure, we herein describe the relevant structural changes in echocardiography at this level. Nevertheless, Tables s1-s3 present both AP and PM level's data and their statistics.

Two weeks post LAD occlusion, untreated rats (control), showed the characteristics of developing LV dysfunction. There was an increase of apical cardiac cross-sectional area at systole (LVESA) to $28.1 \pm 3.2 \mathrm{~mm}^{2}$, $33.4 \pm 3.0 \mathrm{~mm}^{2}, 42.8 \pm 3.2 \mathrm{~mm}^{2}$ and $28.9 \pm 3.5 \mathrm{~mm}^{2}$, in the control, Spironolactone, TUS and Spironolactone + TUS groups, respectively ( $P<0.001$ vs. baseline, for all). Similarly, apical LVEDA enlarged in all groups by $30-100 \%$, reaching $46.4 \pm 3.5 \mathrm{~mm}^{2}, 48.9 \pm 3.2 \mathrm{~mm}^{2}, 62.2 \pm 3.5 \mathrm{~mm}^{2}$ and $41.9 \pm 3.8 \mathrm{~mm}^{2}$ in the control, spironolactone, TUS and spironolactone + TUS groups, respectively $(\mathrm{P}<0.001 \mathrm{vs}$. baseline, for all). Those structural changes attenuated cardiac function, as seen by the diminished EF $40.5 \pm 2.5 \%, 49.5 \pm 2.3 \%, 44.3 \pm 2.5 \%$ and $54.4 \pm 2.7 \%$, in the control, Spironolactone, TUS and Spironolactone + TUS groups, respectively $(P<0.001$ vs. baseline, for all. Table s2).

Four weeks post operation, structurally, the LV was enlarged even more in most groups, thus the AP LVESA was $38.0 \pm 3.2 \mathrm{~mm}^{2}, 44.3 \pm 3.0 \mathrm{~mm}^{2}, 37.9 \pm 3.2 \mathrm{~mm}^{2}$ and $37.7 \pm 3.5 \mathrm{~mm}^{2}$ in the control, Spironolactone, TUS and Spironolactone + TUS groups, respectively $(P<0.001 \mathrm{vs}$. baseline, for all). Likewise, the apical LVEDA were $54.9 \pm 3.5 \mathrm{~mm}^{2}, 62.1 \pm 3.2 \mathrm{~mm}^{2}, 61.1 \pm 3.5 \mathrm{~mm}^{2}$ and $55.3 \pm 3.8 \mathrm{~mm}^{2}$ for the control, Spironolactone, TUS and Spironolactone + TUS groups, respectively $(P<0.001 \mathrm{vs}$. baseline for all). Consequentially, a reduction of $F S$ and $\mathrm{EF}$ post MI were prevented by the spironolactone and spironolactone + TUS treatments. For example, the EF of the control group (untreated group) declined further, reaching only $36.0 \pm 2.5 \%$, which is significantly lower than the EF measured in the spironolactone treated group $(50.1 \pm 2.3 \%, \mathrm{P}<0.05)$, or the spironolactone + TUS (54.0 $\pm 2.7 \%$ ) (Fig. 5 and Table s3).

\section{Gross histology}

Gross histological analysis (Fig. 6) reveals a comparable size of the cardiac muscle in all experimental groups. In the control and TUS specimens, the large scar is occupying 30\% of the LV circumference (Fig. 6A-B). In the specimens taken from the spironolactone and spironolactone + TUS, the scar is less prominent, and the LV walls are thicker and preserved (Fig. 6C-D). Meticulous examination and gross measurements show that the 
average total cardiac area was $78.5 \pm 21.2 \mathrm{~mm}^{2}$, of which LV occupied $63.6 \pm 16.9 \mathrm{~mm}^{2}$, and the LV cavity cross sectional area was $8.0 \pm 4.0 \mathrm{~mm}^{2}$, with no differences among the groups.

\section{Discussion}

Our results show that non-invasive continuous TUS at a frequency of $1 \mathrm{MHz}$ and intensity of $0.5 \mathrm{~W} / \mathrm{cm}^{2}$ exert anti-fibrotic effect when applied early after MI. We found a reduced collagen content in scar regions as well as in remote regions by 2 distinct staining methods as well as by a biochemical method in all treated groups. The anti-fibrotic effect of the treatment was most profound in remote regions from the scar, as can be expected. The reduced fibrosis was associated with reduced pro-collagenous protein expression (TGF- $\beta 1$ ), yet not all treatments (TUS) also affected its downstream effector (smad3). Nevertheless, the combined spironolactone + TUS treatment was effective in reducing both TGF- $\beta 1$ and smad3 expression. Functionally, FS and EF were preserved in spironolactone treated groups, with advantage to the spironolactone + TUS over spironolactone.

Collagen synthesis in humans is initiated as soon as one-week post MI and reaches a steady state at about a month later [22]. Following MI and the subsequent local inflammation, fibroblasts and particularly myocardial fibroblasts express high levels of procollagens of various types. These in turn affect collagen deposition in the newly developed scar [23].

It is postulated that interleukins and cytokines and other inflammatory agents are involved in the fibrotic process [24]. It was shown that an early step after myocardial ischemic injury results with the accumulation of immune cells such as macrophages and lymphocytes through chemokines and adhesion molecules, that eventually cause tissue remodeling and destruction. This is particularly due to the action of cytokines such as interleukins (IL-6, IL-10 and TNF-a) [8]'[17]. Additionally, corticosteroids and mineralocorticoids are produced in the adrenal glands as well as in extra-adrenal organs, including the intestine, skin and possibly the heart [25]. Interestingly, corticosteroids and mineralocorticoids production is also affected by volume status, sodium depletion, RAAS activation and was proven even after adrenalectomy [25]. Thus, as aldosterone, one of those mineralocorticoids, is upregulated after $\mathrm{Ml}$, it may promote cardiac remodeling, myocyte hypertrophy and fibrosis $[1,4,5,20]$.

Finally, TGF- $\beta 1$ is one of the main primary factors that drives fibrosis. The TGF- $\beta$ family is an important mediator of tissue repair [26]. Each TGF- $\beta$ isoform may have a different effect on wound healing. TGF- $\beta 1$ mediates fibrosis in adult wounds [27].

Several studies showed that aldosterone antagonists can reduce collagen deposition, may preserve cardiac function and reduce rats' mortality following MI $[1,4,20]$. They have suggested that the direct beneficiary effect of aldosterone antagonists is mediated through the angiotensin-1 (AT ${ }_{1}$ ) receptor. We evaluated the efficacy of the known anti-fibrotic agent, an aldosterone inhibitor - spironolactone, and the applicability of a spironolactone + TUS combination treatment. Our results are in accordance with the results of previous studies that showed reduced collagen deposition following spironolactone administration in various models of cardiac damage $[1,4,20]$. Further, we are showing an additional relevant mechanism by which spironolactone exerts its antifibrotic effect, i.e., the inhibition of TGF- $\beta 1 /$ smad3 pathway. 
In our study, we applied TUS shortly after ligation of the LAD artery for 28 consecutive days, and we tested its anti-fibrotic efficacy. The results suggest a positive effect of the TUS treatment at remote collagen deposition reduction (CVF and hydroxyproline) as well as inhibiting TGF- $\beta 1$ expression, while failing to reduce smad3 expression, or to achieve a significant physiological efficacy.

Currently, two major aspects impede the TUS full efficacy potential. First is the assembly of the correct TUS profile, and second is the understanding of its mechanism of action. The TUS profile and effect is determined by numerous parameters, among them the frequency, intensity, duty cycle (DC), pulse repetition frequency (PRF), duration of each session, timing of treatment administration (when after damage, how many sessions), etc. Each might hamper the treatment efficacy. The second aspect, i.e., TUS's mechanism of action is still very much obscure. It was established that TUS affects tissues through either thermal or non-thermal mechanisms [28]. At the cellular and the vascular levels, TUS augments blood supply and depresses tissue remodeling [29]. Studies demonstrate that TUS affects cytokines, fibroblast growth factors, collagen and phagocytosis of macrophages and other immune cells which cumulate to depressed phagocytic index in-vivo [29]. In another invivo setup, TUS induced endothelial dependent vasodilation [30]. Specifically, in that study, canine coronaries and human femoropopliteal arteries underwent vasodilation by TUS, which resulted in increased tissue oxygenation [30]. Another important effect of TUS is its pro-angiogenic effect. Young and Dyson showed that injured tissues exposed to TUS had more blood vessels in their granulation tissue [31].

The positive effect of the TUS can thus be explained by the vasodilatory as well as the pro-angiogenic effect of TUS, which may improve the oxygenation status of the damaged cardiac muscle following MI. Together with its anti-inflammatory effect, it may induce preservation of cardiac muscle and inhibition of its replacement by collagen. Although the TUS anti-fibrotic mechanism is still unknown, one can postulate that both treatments (spironolactone and TUS) may have complementary effects. The anti-aldosterone treatment, which exerts its effect through $\mathrm{AT}_{1}$ receptor, may further inhibit collagen deposition, and TUS, by its effects, may also contribute to the distribution of spironolactone in damaged tissues, such as the scar and its surroundings. By that, TUS can enhance the effect of spironolactone, as well as induce its own beneficiary effects.

Finally, the current study shed some light regarding both TUS's impeding aspects. We have clearly seen significant and relevant biological effect of the selected TUS profile. Yet, further research is needed to fine-tune the TUS profile in order to achieve significant physiological efficacy.

In our study, we did observe a significant reduction in TGF- $\beta 1$ expression in all treated groups compared to the control group, which confirms our hypothesis and verifies previous results that cytokines play a role in collagen synthesis and cardiac fibrosis [8]. Smad3 is an essential component in the signaling pathway of TGF- $\beta$, which rests downstream to the TGF- $\beta$ receptors [32]. Lately, we showed that empagliflozin, a sodium/glucose transporter 2 inhibitor, also attenuate the TGF- $\beta 1 /$ smad3 pathway and prevent post-MI fibrosis and preserves cardiac structure [10]. Here, we show that spironolactone, but not TUS, affects the downstream components of this pro-fibrotic pathway, smad3 [32]. This raises the question whether TUS can affect biochemical fibrotic pathways? Rossato et al. showed more of the anti-inflammatory effects of TUS, as it increases antiinflammatory cytokines expression (IL-10) and decreases pro-inflammatory cytokines (IL-6 and TNFa) [8]. Additionally, the involvement of TGF- $\beta 1$ and active phosphorylated smad3 ( $p$-smad3) was shown previously by Guo et al., who showed that both TGF- $\beta 1$ its downstream active p-smad3 are affected by a physical treatment, 
i.e., SDT (Guo et al., 2016). Collectively, these data may hint that TUS has the potential to affect fibrotic pathways. Accordingly, modified TUS profile may also complete the effect on the TGF- $\beta 1 /$ smad3 pathway, and we cannot exclude the possibility that the current TUS treatment has affected IL-10, IL-6 or TNFa, as they were not tested in the current study. Nevertheless, further research is needed to accomplish physiological efficacy.

\section{Study limitation}

In the current research, we used a specific TUS protocol, which is characterized by its frequency, intensity, number and duration of treatments. Each parameter may be altered and may change the efficacy of the TUS treatment. Moreover, other parameters, which are not included here may modify the TUS profile and subsequently its biological effect. Among those are the DC and PRF, as well as the total number of treatments.

\section{Conclusions}

The data presented here show that TUS, spironolactone and their combination reduce collagen content and fibrosis post MI. This physiological effect may further affect LV remodeling and prevent heart failure in the long-term. As TUS is still a rather new entity in the medical arena, further research is essential in order to find the optimal TUS profile and its additive effect to pharmacological treatments, in order to modify LV remodeling. The results of our research should encourage additional studies in this direction.

\section{Abbreviations}

Myocardial infarction: MI; Therapeutic ultrasound: TUS; Transforming growth factor $\beta 1$ : TGF- $\beta 1$; Small mother against decapentaplegic 3: smad3; Left ventricular: LV; Renin-angiotensin-aldosterone system: RAAS; Nitric oxide synthase: NOS; Nitric oxide: NO; Sonodynamic therapy: SDT; Aminolevulinic acid: ALA; Apical: AP; Papillary muscle: PM; Two-dimensional: 2D; Fractional shortening: FS; LV intra-ventricular diameters at systole: LVIDs; LV intra-ventricular diameters at diastole: LVIDd; Fractional area change: FAC; LV end diastolic area: LVEDA; LV end systolic area: LVESA; Ejection fraction: EF; Left anterior descending: LAD; Hematoxylin and Eosin: H\&E; Collagen volume fraction: CVF; Analysis of variance: ANOVA; Interleukin: IL; Angiotensin-1: AT ; Duty cycle: DC; Pulse repetition frequency: PRF;

\section{Declarations}

\section{Authors' contributions}

AS devised the research and was a major contributor to writing the manuscript. ED and OE performed the animal surgeries, obtained, analyzed and interpreted chemical and histological data, performed the statistical analyses and were contributors to writing the manuscript. AR and TR obtained the physiological data, GM and JA performed manuscript review. All authors read and approved the final manuscript.

\section{Funding}

This study was not funded by any grant.

\section{Availability of data and materials}


The datasets used and analyzed during the current study are available from the corresponding author on reasonable request.

\section{Ethical approval}

All applicable international, national, and/or institutional guidelines for the care and use of animals were followed (see first paragraph in method section).

\section{Consent for publication}

Not Applicable.

\section{Conflict of interest}

The authors declare that they have no conflict of interests.

\section{References}

1. Mill JG, Milanez MDC, De Resende MM, Gomes MDGS, Leite CM. Spironolactone prevents cardiac collagen proliferation after myocardial infarction in rats. Clin Exp Pharmacol Physiol. 2003;30(10):739-44.

2. Delcayre C, Silvestre JS, Garnier A, Oubenaissa A, Cailmail S, Tatara E, et al. Cardiac aldosterone production and ventricular remodeling. In: Kidney International. 2000. p. 1346-51.

3. Silvestre JS, Heymes C, Oubénaïssa A, Robert V, Aupetit-Faisant B, Carayon A, et al. Activation of cardiac aldosterone. Circulation. 1999;99(20):2694-701.

4. Brilla CG, Matsubara LS, Weber KT. Antifibrotic effects of spironolactone in preventing myocardial fibrosis in systemic arterial hypertension. Am J Cardiol. 1993;71(3):12(A)-16(A).

5. Campbell SE, Janicki JS, Matsubara BB, Weber KT. Myocardial fibrosis in the rat with mineralocorticoid excess prevention of scarring by amiloride. Am J Hypertens. 1993;6(6):487-95.

6. Leifheit-Nestler M, Kirchhoff F, Nespor J, Richter B, Soetje B, Klintschar M, et al. Fibroblast growth factor 23 is induced by an activated renin-angiotensin-aldosterone system in cardiac myocytes and promotes the pro-fibrotic crosstalk between cardiac myocytes and fibroblasts. Nephrol Dial Transplant. 2018;33(10):1722-34.

7. Sztechman D, Czarzasta K, Cudnoch-Jedrzejewska A, Szczepanska-Sadowska E, Zera T. Aldosterone and mineralocorticoid receptors in regulation of the cardiovascular system and pathological remodelling of the heart and arteries. Vol. 69, Journal of Physiology and Pharmacology. 2018. p. 829-45.

8. Rossato DD, Lago PD, Hentschke VS, Rucatti AL, Signori LU, Silveira MN, et al. Ultrasound modulates skeletal muscle cytokine levels in rats with heart failure. Ultrasound Med Biol. 2015;41(3):797-805.

9. Kang S, Verma S, Hassanabad AF, Teng G, Belke DD, Dundas JA, et al. Direct Effects of Empagliflozin on Extracellular Matrix Remodelling in Human Cardiac Myofibroblasts: Novel Translational Clues to Explain EMPA-REG OUTCOME Results. Can J Cardiol. 2020;36(4):543-53.

10. Daud E, Ertracht O, Bandel N, Moady G, Shehadeh M, Reuveni T, et al. The impact of empagliflozin on cardiac physiology and fibrosis early after myocardial infarction in non-diabetic rats. Cardiovasc Diabetol [Internet]. 2021;20(1). Available from: https://doi.org/10.1186/s12933-021-01322-6 
11. Tzou DT, Usawachintachit M, Taguchi K, Chi T. Ultrasound use in urinary stones: Adapting old technology for a modern-day disease. J Endourol. 2017;31:S89-94.

12. Wan GY, Liu Y, Chen BW, Liu YY, Wang YS, Zhang N. Recent advances of sonodynamic therapy in cancer treatment. Vol. 13, Cancer Biology and Medicine. 2016. p. 325-38.

13. Barzelai S, Sharabani-Yosef O, Holbova R, Castel D, Walden R, Engelberg S, et al. Low-intensity ultrasound induces angiogenesis in rat hind-limb ischemia. Ultrasound Med Biol. 2006 Jan;32(1):139-45.

14. Huang JJ, Shi YQ, Li RL, Hu A, Zhou HS, Cheng Q, et al. Angiogenesis effect of therapeutic ultrasound on ischemic hind limb in mice. Am J Transl Res. 2014;6(6):703-13.

15. Altland OD, Dalecki D, Suchkova VN, Francis CW. Low-intensity ultrasound increases endothelial cell nitric oxide synthase activity and nitric oxide synthesis. J Thromb Haemost. 2004;2(4):637-43.

16. Atar S, Siegel RJ, Akel R, Ye Y, Lin Y, Modi S a, et al. Ultrasound at 27 kHz Increases Tissue Expression and Activity of Nitric Oxide Synthases in Acute Limb Ischemia in Rabbits. Ultrasound Med Biol. 2007 Sep;33(9):1483-8.

17. Guo Y, Dong Z, Shi Y, Wang W, Wang L, Sun J, et al. Sonodynamic Therapy Inhibits Fibrogenesis in Rat Cardiac Fibroblasts Induced by TGF- $\beta 1$. Cell Physiol Biochem. 2016;40(3-4):579-88.

18. Farcic TS, Baldan CS, Cattapan CG, Parizotto NA, João SMA, Casarotto RA. Treatment time of ultrasound therapy interferes with the organization of collagen fibers in rat tendons. Brazilian J Phys Ther. 2013;17(3):263-71.

19. Blumberg Y, Ertracht O, Gershon I, Bachner-Hinenzon N, Reuveni T, Atar S. High-Intensity Training Improves Global and Segmental Strains in Severe Congestive Heart Failure. J Card Fail [Internet]. 2017;23(5):392402. Available from: http://dx.doi.org/10.1016/j.cardfail.2016.12.010

20. Cezar MDM, Damatto RL, Pagan LU, Lima ARR, Martinez PF, Bonomo C, et al. Early Spironolactone Treatment Attenuates Heart Failure Development by Improving Myocardial Function and Reducing Fibrosis in Spontaneously Hypertensive Rats. Cell Physiol Biochem. 2015;36(4):1453-66.

21. Reddy GK, Enwemeka CS. A simplified method for the analysis of hydroxyproline in biological tissues. Clin Biochem. 1996;29(3):225-9.

22. Richardson WJ, Clarke SA, Alexander Quinn T, Holmes JW. Physiological implications of myocardial scar structure. Compr Physiol. 2015;5(4):1877-909.

23. Daskalopoulos EP, Janssen BJA, Blankesteijn WM. Myofibroblasts in the infarct area: Concepts and challenges. Microsc Microanal. 2012;18(1):35-49.

24. Puhakka M, Magga J, Hietakorpi S, Penttilä I, Uusimaa P, Risteli J, et al. Interleukin-6 and tumor necrosis factor alpha in relation to myocardial infarct size and collagen formation. J Card Fail. 2003;9(4):325-32.

25. Taves MD, Gomez-Sanchez CE, Soma KK. Extra-adrenal glucocorticoids and mineralocorticoids: Evidence for local synthesis, regulation, and function. Vol. 301, American Journal of Physiology - Endocrinology and Metabolism. 2011. p. E11-24.

26. Penn JW, Grobbelaar AO, Rolfe KJ. The role of the TGF- $\beta$ family in wound healing, burns and scarring: a review. Int J Burns Trauma [Internet]. 2012;2(1):18-28. Available from: http://www.ncbi.nlm.nih.gov/pubmed/22928164\%0Ahttp://www.pubmedcentral.nih.gov/articlerender.fcgi? artid=PMC3415964 
27. Lichtman MK, Otero-Vinas M, Falanga V. Transforming growth factor beta (TGF- $\beta$ ) isoforms in wound healing and fibrosis. Wound Repair Regen. 2016;24(2):215-22.

28. Johns LD. Nonthermal effects of therapeutic ultrasound: The frequency resonance hypothesis. Vol. 37, Journal of Athletic Training. 2002. p. 293-9.

29. Anderson DW, Barrett JT. Depression of phagocytosis by ultrasound. Ultrasound Med Biol. 1981;7(3):26773.

30. Steffen W, Cumberland D, Gaines P, Luo H, Nita H, Maurer G, et al. Chrome congestive heart failure: Catheter-delivered high intensity, low frequency ultrasound induces vasodilation in vivo. Eur Heart $\mathrm{J}$. 1994;15(3):369-76.

31. Young SR, Dyson M. The effect of therapeutic ultrasound on angiogenesis. Ultrasound Med Biol. 1990;16(3):261-9.

32. Bujak M, Frangogiannis NG. The role of TGF- $\beta$ signaling in myocardial infarction and cardiac remodeling. Vol. 74, Cardiovascular Research. 2007. p. 184-95.

33. Cai J, Chen X, Chen X, Chen L, Zheng G, Zhou H, et al. Anti-Fibrosis Effect of Relaxin and Spironolactone Combined on Isoprenaline-Induced Myocardial Fibrosis in Rats via Inhibition of Endothelial-Mesenchymal Transition. Cell Physiol Biochem. 2017;41(3):1167-78.

\section{Figures}



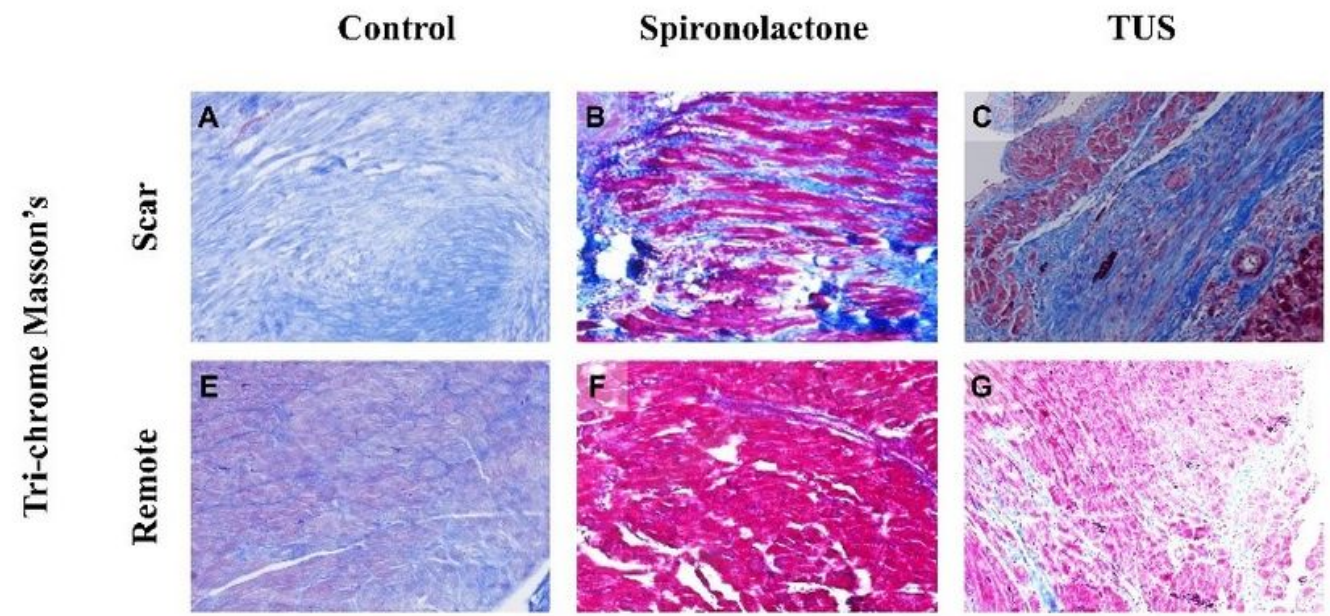

Spironolactone + TUS


\section{Figure 1}

Representative histological specimens of cardiac muscles: first and second rows stained with Tri- chrome Masson's, third and fourth rows are Picro-Sirius red stained. Specimens in the first and third rows were taken from scar areas, and second and fourth rows were taken from remote areas. Each column represents the designated treatment. (x100 magnification). 
A
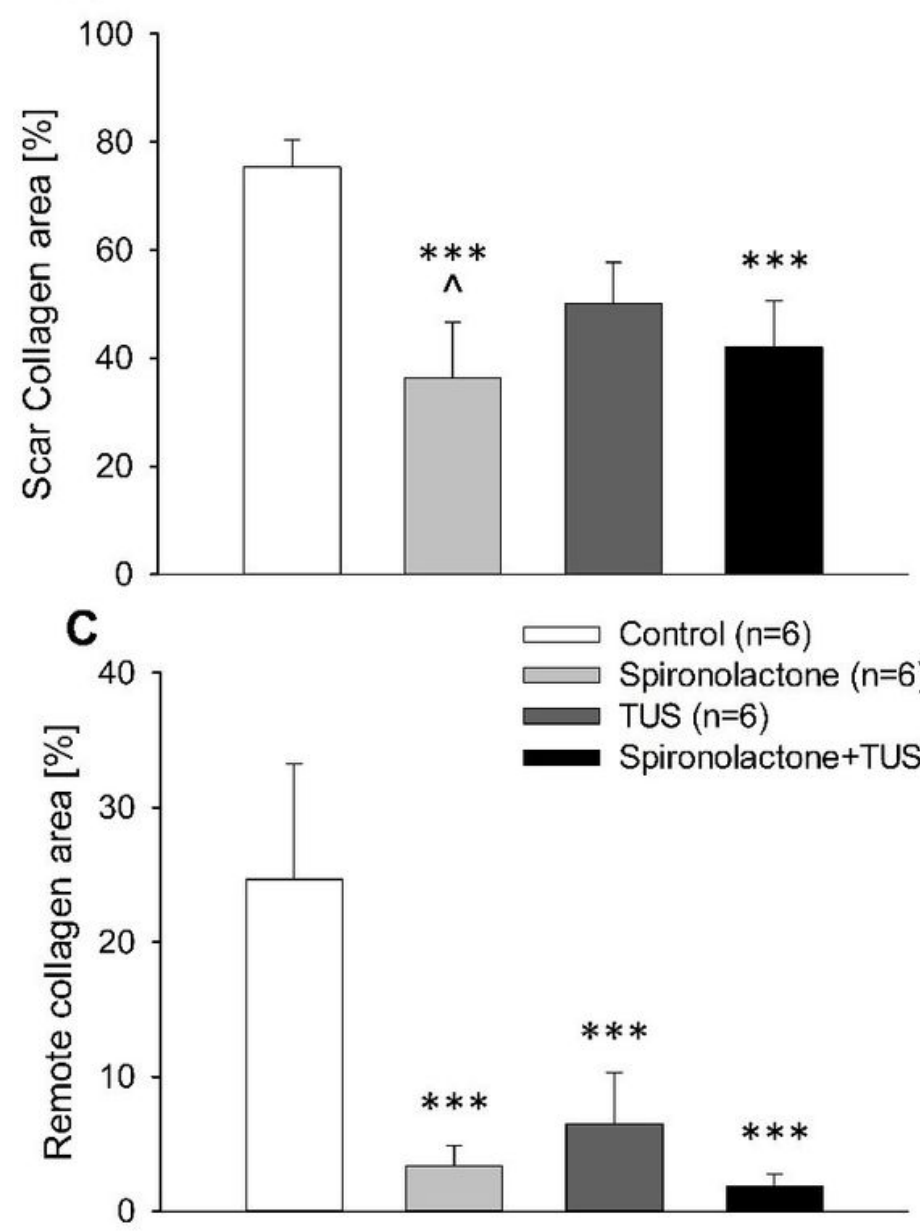

Tri-chrome Masson's
B
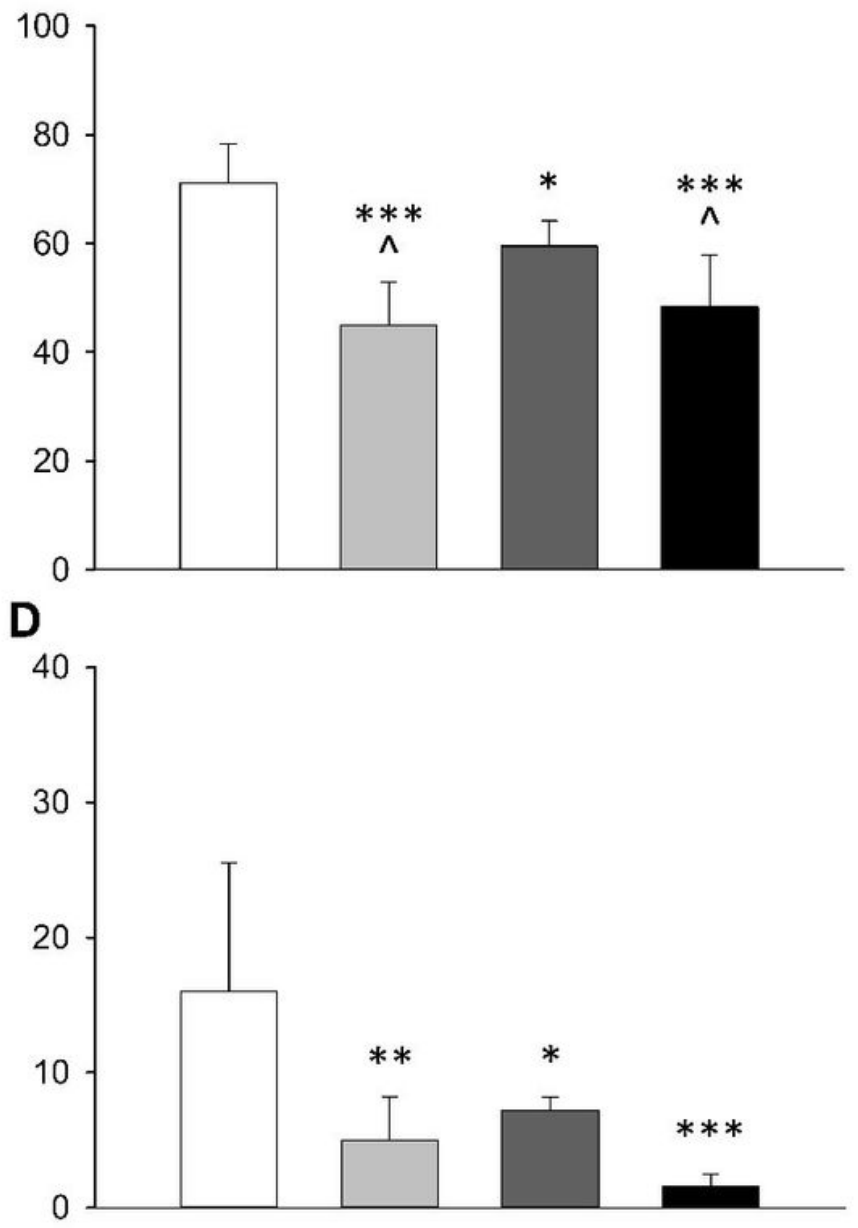

Picro-Sirius red

Figure 2

Quantification of collagen volume fraction in specimens: (A) taken from remote areas and (B) scar areas. Left panel quantification from Tri-chrome Masson's stained specimens. Right panel quantification from Picro-Sirius red stained specimens. ${ }^{*} \mathrm{P}<0.05$ vs. control, ${ }^{* *} \mathrm{P}<0.01$ vs. control, ${ }^{* \star *} \mathrm{P}<0.001$ vs. control, ${ }^{\wedge} \mathrm{P}<0.05$ vs. TUS. 

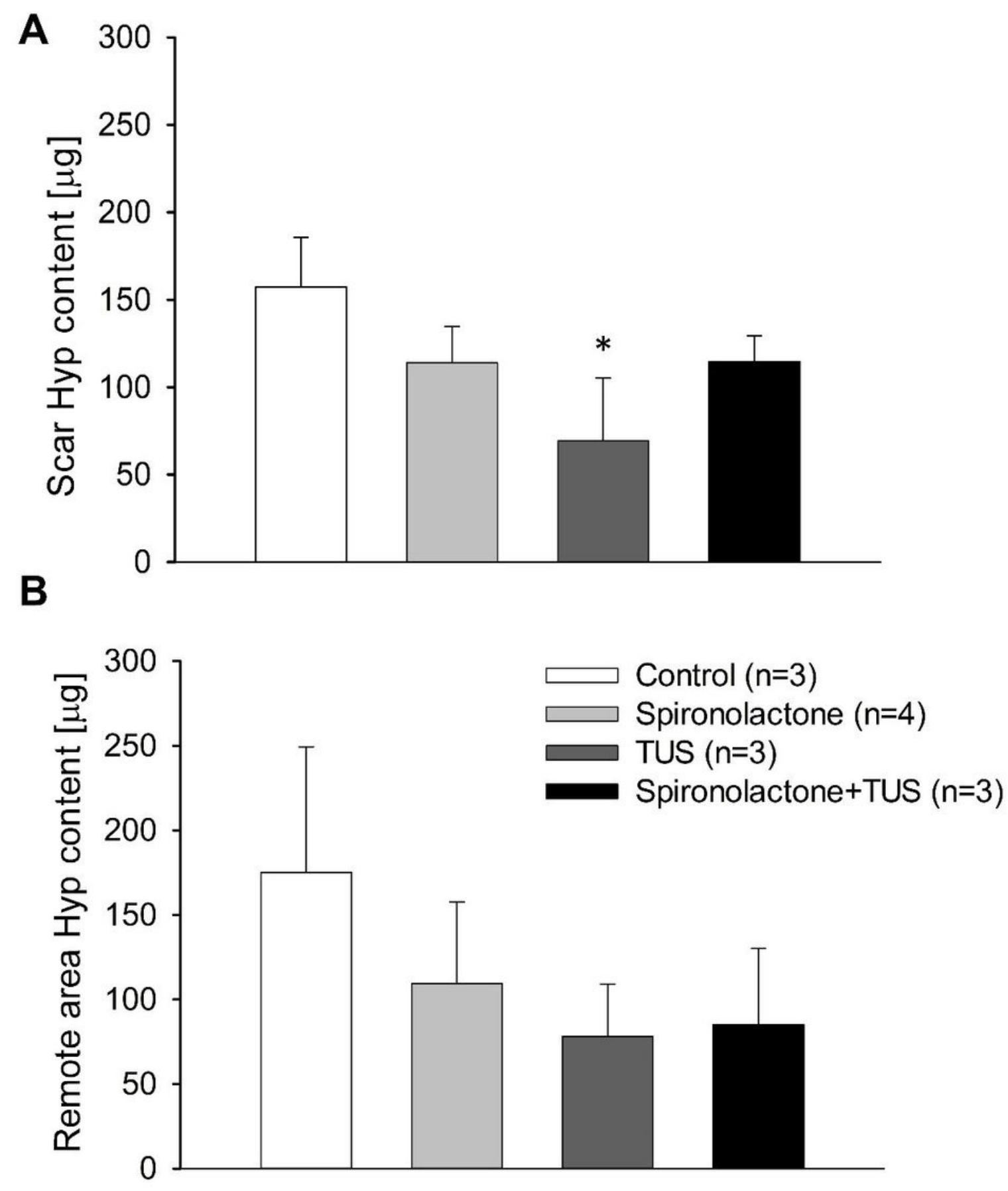

Figure 3

Hydroxyproline analysis: (A) hydroxyproline content in specimens taken from remote areas of the different experimental groups, (B) hydroxyproline content in specimens taken from scars of the different experimental groups. ${ }^{*} \mathrm{P}<0.05$ vs. control. 

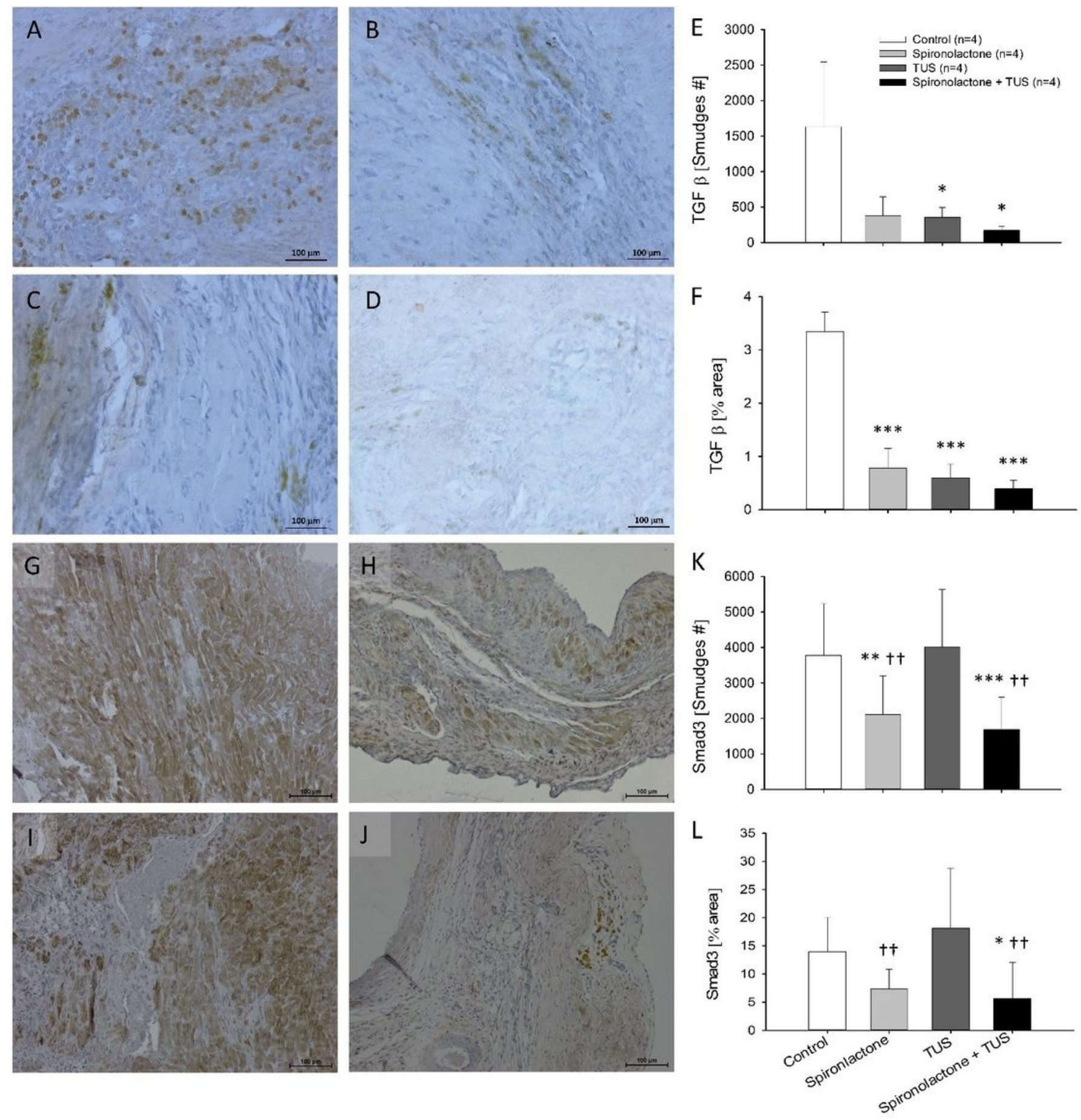

\section{Figure 4}

TGF- $\beta 1$ and Smad3 expressions are attenuated by TUS, spironolactone and spironolactone + TUS. (A-D) Representative immunohistochemical staining of TGF- $\beta 1$ (magnification $\times 100$ ). (E) Quantification of number of TGF- $\beta 1$ smudges and (F) \% area occupied by TGF- $\beta 1$ at the scar section of the cardiac muscle in all experimental groups. (G-J) Representative immunohistochemical staining of Smad3 (magnification $\times 100)$. (K) Quantification of number of Smad3 smudges and (I) \% area occupied by Smad3 at the scar section of the cardiac muscle in all experimental groups. ${ }^{*} \mathrm{P}<0.05$ vs. Control, $* \star \mathrm{P}<0.01$ vs. Control, ${ }^{\star \star \star} \mathrm{P}<0.001$ vs. Control, †† $\mathrm{P}<0.01$ vs. TUS. 

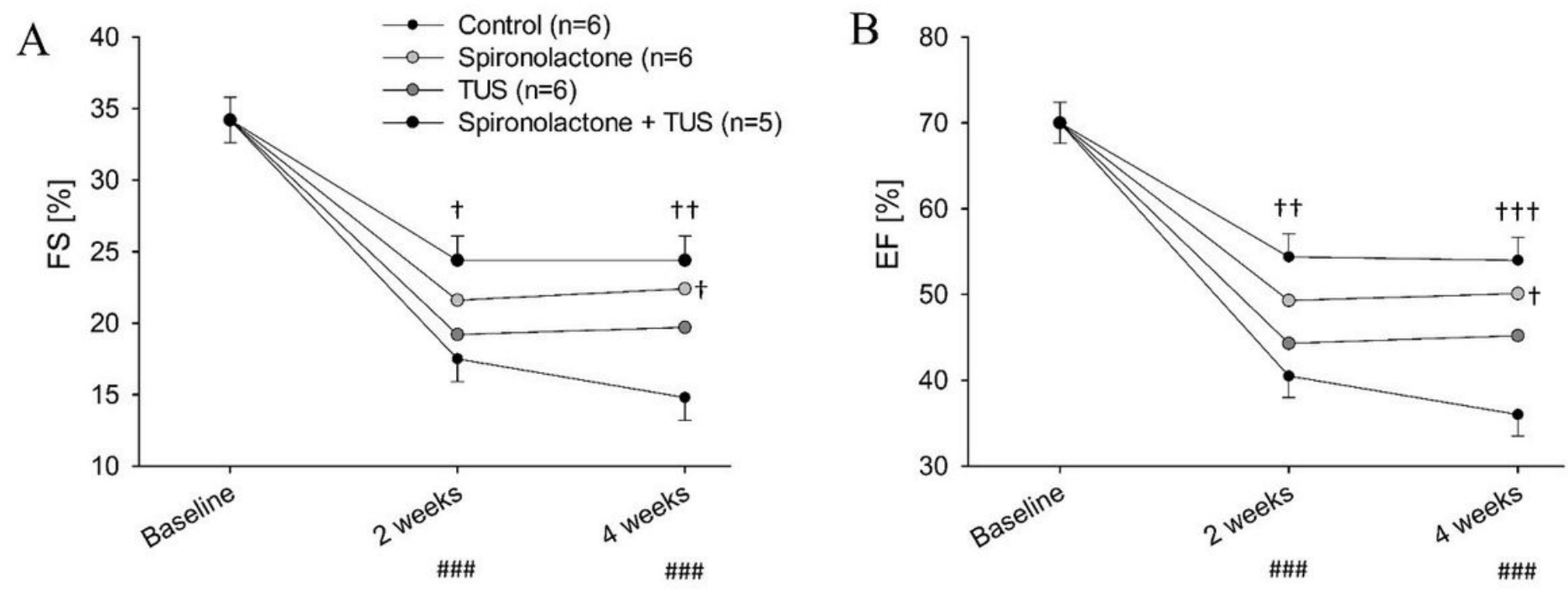

\section{Figure 5}

Echocardiographic functional parameters A. FS and B. EF at the different time points. $\dagger \mathrm{P}<0.05 \mathrm{vs}$. control, $\uparrow \dagger$ $\mathrm{P}<0.01$ vs. control, $+\dagger \dagger \mathrm{P}<0.001$ vs. control, \#\#\# $\mathrm{P}<0.001$ vs. baseline reading. 

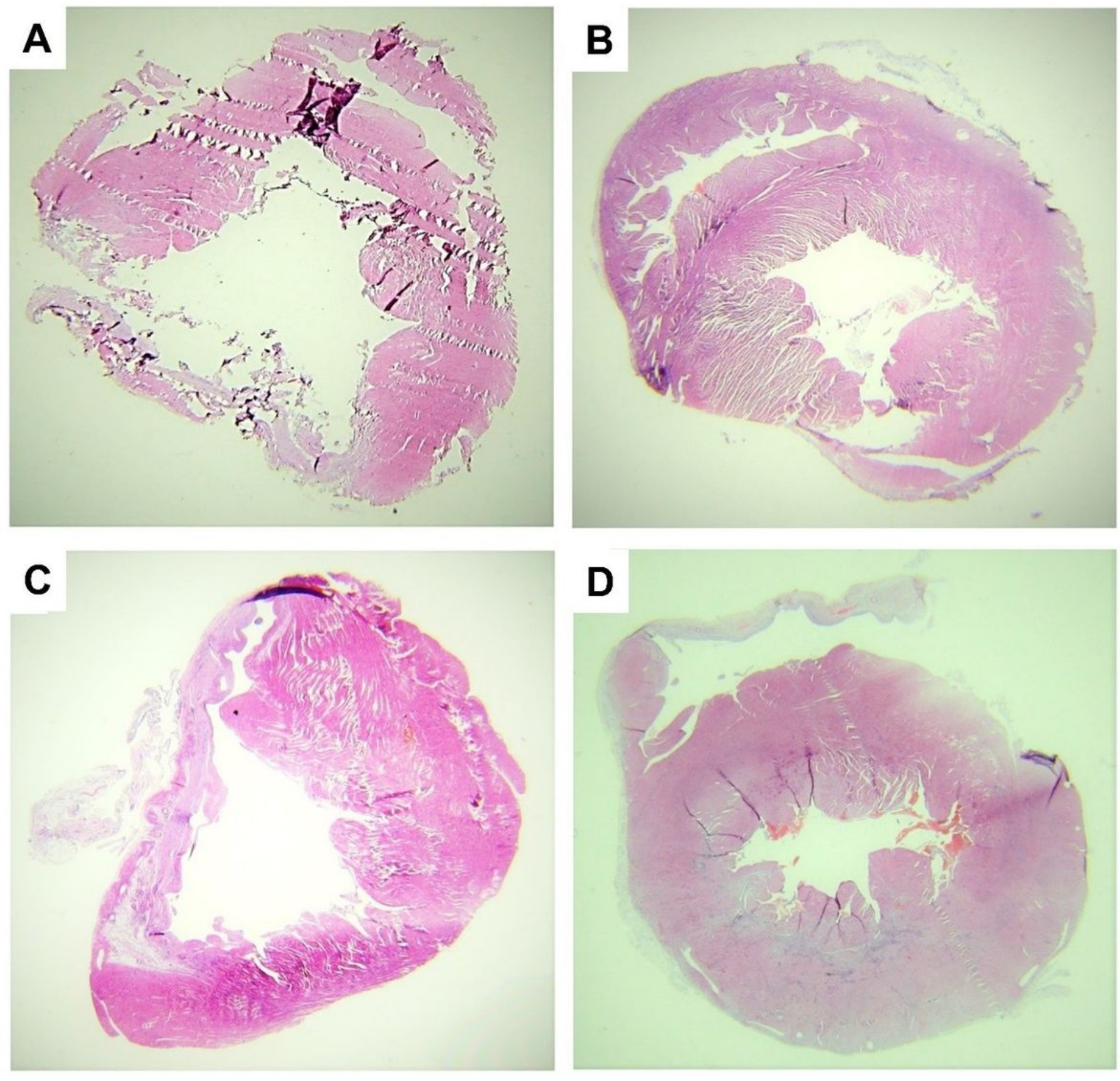

\section{Figure 6}

Representative cardiac histological sections at the PM level: (A) Control (untreated rat), (B) Spironolactone treated rat, (C) TUS treated rat and (D) Spironolactone + TUS treated rat.

\section{Supplementary Files}

This is a list of supplementary files associated with this preprint. Click to download.

- SupplementaryTablesTUSandfibrosispostMlinrats.docx 